\title{
Downstream evolution of unconfined vortices: mechanical and thermal aspects
}

\author{
By M. PÉREZ-SABORID, M. A. HER RADA, \\ A. GÓMEZ-BAREA AND A. BARRERO \\ Escuela Superior de Ingenieros, Universidad de Sevilla, 41092 Sevilla, Spain
}

(Received 5 September 2000 and in revised form 31 May 2002)

\begin{abstract}
We present a numerical study of the downstream evolution (mechanical and thermal) of vortex-jet cores whose velocity and temperature fields far from the axis match a family of inviscid and non-conducting vortices. The far-velocity field is rotational, except for a particular case which corresponds to the well-known Long's vortex. The evolution of the vortex core depends on both the conditions at a certain upstream station, characterized by the dimensionless value of the velocity at the axis, and a dimensionless swirling parameter $L$ defined as the ratio of the values of the azimuthal and axial velocities outside the vortex core. This numerical study, based on the quasi-cylindrical approximation (QC) of the Navier-Stokes equations, determines the conditions under which the vortex evolution proceeds smoothly, eventually reaching an asymptotic self-similar behaviour as described in the literature (Fernández-Feria, Fernández de la Mora \& Barrero 1995; Herrada, Pérez-Saborid \& Barrero 1999), or breaks in a non-slender solution (vortex breakdown). In particular, the critical value $L=L_{b}(a)$ beyond which vortex breakdown occurs downstream is a function of a dimensionless parameter $a$ characterizing the axial momentum of the vortex jet at an initial upstream station. It is found numerically that for very large values of $a$ this vortex breakdown criterion tends to an asymptote which is precisely the value $L=L^{*}$ predicted by the self-similar analysis, and beyond which a self-similar structure of the vortex core does not exist. In addition, the computation of the total temperature field provides useful information on the physical mechanisms responsible for the thermal separation phenomenon observed in Ranque-Hilsch tubes and other swirling jet devices. In particular, the mechanical work of viscous forces which gives rise to an intense loss of kinetic energy during the initial stages of the evolution has been identified as the physical mechanism responsible for thermal separation.
\end{abstract}

\section{Introduction}

Swirling flows at large Reynolds numbers exhibit a number of distinctive features, which are not yet sufficiently well understood. An example is the phenomenon of vortex breakdown characterized by an abrupt structural change in the flow of a vortex core, which consists of a sudden deceleration of the axial flow and the formation of a stagnation zone followed by increased levels of steadiness and turbulence (see classical works by Leibovich 1984; Escudier 1988; Sarpkaya 1971, 1995 among others). Several breakdown patterns ranging from asymmetric spiral waves to almost axisymmetric bubbles have been observed. The prediction and control of this phenomenon is relevant to several physical and engineering applications such as tornadoes, delta wings at high angles of attack, swirling flows inside pipes, combustion chambers, 
hydro-cyclone separators, etc. Another striking feature which swirling flows often exhibit is that of thermal energy separation (the Ranque-Hilsch effect), which is characterized by a substantial decrease of the stagnation temperature at the vortex axis.

In this paper, we restrict ourselves to the study of free swirling flows such as tornadoes. The cases of vortex flows in pipes or rotating flows in closed containers are not considered here. Among the most relevant theoretical works on free swirling flows is that due to Long (1961), who studied the structure of a self-similar viscous vortex core driven by an outer axisymmetric irrotational flow. Far from the core, the axial and the azimuthal components of the velocity decay as the inverse of the distance to the axis. Long characterized the different near-axis viscous solutions by means of the flow force $M$, a parameter related to the dimensionless axial momentum flux of the flow, which is independent of the axial coordinate. Long found that for values of $M$ smaller than a critical value $M^{*}$ no self-similar solution exists, while for $M>M^{*}$ there exist two different solutions which were termed types I and II by Burggraf \& Foster (1977).

Burggraf \& Foster (1977) solved numerically the quasi-cylindrical (QC) equations for the downstream evolution of a viscous vortex core whose axial and azimuthal velocity components decay far from the axis as $r^{-1}$, where $r$ is the distance to the axis. A Burger's jet-like profile was assumed to exist at the upstream station of the axial coordinate $(z=0)$. The numerical results showed that for values of the flow force $M$ (fixed by the conditions at the upstream station) larger than the critical value $M^{*}$, the vortex core evolves towards one of the two Long's self-similar solutions, while for $M<M^{*}$ the QC approximation breaks down at a certain distance from the upstream station and no self-similar solution is reached, just as predicted by Long. Following Hall (1972), Burggraf \& Foster interpreted the failure of the QC equations to produce self-similar solutions as vortex breakdown.

A different approach to vortex breakdown was pioneered by Benjamin (1962) who established an analogy between vortex breakdown and the hydraulic jump in which, as is well known, a flow undergoes a supercritical-subcritical transition. Hall's (viscous) and Benjamin's (inviscid) approaches to vortex breakdown are not contradictory in principle. However, we will present in the Appendix a family of non-viscous conical vortex flows that do not exhibit vortex breakdown when the mathematical scenario of Benjamin's criterion is applied. Only when viscosity effects are included to regularize the vortex core does this family of vortices present vortex breakdown for finite values of the swirl strength.

In the case of conically similar flows, Shtern \& Hussain (1996) have clarified the above picture. These authors found the existence of a third branch (type III) of selfsimilar solutions to the Navier-Stokes equations, which forms a hysteretic loop with the other two branches. Jump transitions between the different branches are found when the control parameter of the flow reaches one or more threshold values. For high Reynolds numbers, the near-axis behaviour of two of these branches corresponds to Long's solutions of type I and II, while the third one (type III) corresponds to a two-cell flow. In this case, an inviscid, but rotational, outer flow and a potential, non-slender, inner flow are separated by a viscous conical fan jet. Shtern \& Hussain found that at $M=M^{*}$, with decreasing $M$, a solution of type I jumps to a solution of type III. They related this phenomenon to vortex breakdown since the two-cell structure in conical flows bears a strong resemblance to the bubble structure with re-circulating motion and negligible swirl inside that results very often from the vortex breakdown phenomenon. 
The analysis of the Long vortex was extended by Fernández-Feria, Fernández de la Mora \& Barrero (1995) who considered self-similar swirling vortex cores which asymptotically match Euler solutions whose axial and azimuthal velocity components behave near the axis as $r^{m-2}$, where $r$ is the radial distance and $0<m<2$; the case $m=1$ corresponds to Long vortex. The physical relevance of the flows analysed by Fernández-Feria and co-workers, especially those with $1<m<2$, appears when one considers the large amount of experimental data (Keller, Egli \& Exley 1985; Vatistas, Lin \& Kwok 1986; Ogawa 1993) which suggest that the azimuthal velocity of the inviscid flow around real vortex cores is not exactly irrotational $(m=1)$ but of the form $r^{-n}$ with power $n$ in general smaller than 1 ( $m$ larger than 1). In particular, Emanuel (1991) has pointed out that outside the core of a hurricane the winds fall off gradually with radius, obeying approximately an $r^{-1 / 2}$ law, corresponding just to $m=1.5$. In addition, the flows considered by Fernández-Feria et al. (1995) reproduce some of the observed features of real vortex flows, i.e. vortex breakdown. These flows can be characterized by an inviscid dimensionless swirling parameter $L$, the Squire parameter, defined as the ratio between the azimuthal $v$ and axial $w$ components of the inviscid velocity near the axis:

$$
v=L W r^{m-2}, \quad w=W r^{m-2},
$$

where $W$ is a dimensional constant. The pressure field is given by Bernouilli's equation and near the axis behaves as

$$
\frac{p}{\rho}=\frac{p_{r}}{\rho}+\frac{(L W)^{2}}{2(m-2)} r^{2(m-2)},
$$

$p_{r}$ being a constant reference pressure. Parameter $L$ plays a role somewhat analogous to parameter $M$ for Long's vortex: when $1<m<2$ no solutions exist for values of $L$ larger than a critical value $L^{*}(m)$, which depends on $m$, while two self-similar solutions (types I and II) are possible for $L<L^{*}(m)$. This catastrophic behaviour which occurs beyond $L^{*}$ was interpreted as vortex breakdown. For the case $m=1$ (Long vortex), the outer flow and the near-axis boundary layer match only for the particular value $L=\sqrt{2}$ (Long 1961; Fernández-Feria et al. 1995), so that, in this case, the azimuthal and meridian motions are coupled and parameter $L$ is no longer suitable for the description of the $m=1$ flows. In addition, for $m \neq 1$, the existence of a third branch (type III) in conically similar flows at high Reynolds numbers has also been found (Fernández-Feria et al. 1999). For $1<m<2$ and $L=L^{*}$, with increasing $L$ a solution of type I jumps to a solution of type III where an irrotational inner cell is separated from the inviscid but rotational outer cell by a very thin viscous conical fan jet. The main conclusion of this study using self-similar solutions is that the singularities often appearing in axisymmetric inviscid swirling flows cannot always be regularized through thin viscous layers. As a result, a sharp boundary is established between some physically unacceptable inviscid flows and those whose existence is not forbidden by viscosity. These authors argued that the non-existence of analytical solutions of slender self-similar viscous cores beyond a threshold value supports vortex breakdown as a phenomenon of boundary layer separation type.

The success of the self-similar conical solutions in explaining some mechanical features of the structure of vortex cores motivated Herrada, Pérez-Saborid \& Barrero (1999) to extend this model to account for thermal effects. In particular, they used the results obtained by Fernández-Feria et al. (1995) to analyse the thermal structure of self-similar vortex cores in situations where compressibility effects are small. Herrada and coworkers found that this family of self-similar vortices exhibits a substantial 
decrease of the stagnation temperature (Ranque-Hilsch effect) at the vortex axis. It should be emphasized that this thermal structure is obtained with a self-consistent and physically realistic model without the need to resort to previous ad hoc explanations such as turbulent heat transport or unsteady (acoustic) effects, see for example Deissler $\&$ Perlmutter (1960) and Kurosaka (1982).

In this analysis, Herrada and co-workers assumed a viscous and conducting gas vortex core driven by an inviscid and non-conducting gas outer vortex whose velocity, pressure, and temperature fields satisfy the Euler equations. They assumed small relative density variations in the flow (quasi-incompressible flow), whose order of magnitude is related to those of pressure and temperature, according to the perfect gas law, by $\Delta \rho / \rho \sim \Delta p / p-\Delta T / T$. Observe that small values of the relative pressure variations are equivalent to small values of the flow Mach number since, according to the momentum equation, $\Delta p / p \sim \rho v^{2} / p \sim M^{2}$. Since the mechanical work of the pressure forces is the leading term in the energy equation, it is easily shown that the temperature changes in the flow $\Delta T / T$ are also $O\left(M^{2}\right)$. Therefore, for a perfect gas, the density changes are also $O\left(M^{2}\right)$. For sufficiently small $M$ the changes in the thermodynamic properties from a base thermodynamic state are much smaller than the velocity component which is $O(M)$. Specifically, in a such a limit, the continuity equation reduces in the leading order $O(M)$ to the incompressible flow equation $\nabla \cdot v=0$. Then, the leading-order momentum equations completely determine the pressure and velocity fields. Finally, the temperature field can be computed from the known pressure and velocity fields using the leading-order energy equation. This is just the quasi-incompressible approximation of the Euler (or Navier-Stokes) equations in which the mechanical problem (continuity and momentum equations) can be solved independently of the thermal problem in the absence of buoyancy effects.

Then, Herrada and coworkers calculated the temperature field for the inviscid and non-conducting outer flow using entropy conservation along streamlines. In effect, for a perfect gas

$$
S-S_{r}=c_{p} \ln \frac{T / T_{r}}{\left(p / p_{r}\right)^{(\gamma-1) / \gamma}},
$$

where $S_{r}$ and $T_{r}$ are constant reference entropy and temperature respectively, $p_{r}=$ $\rho R_{g} T_{r}$, and $c_{p}$ and $R_{g}$ are the specific heat ratio at constant pressure and the gas constant respectively. For $\left(T-T_{r}\right) / T_{r}$ and $\left(p-p_{r}\right) / p_{r}$ small enough (1.3) leads to

$$
c_{p}\left[\frac{T-T_{r}}{T_{r}}-\frac{\gamma-1}{\gamma} \frac{p-p_{r}}{p_{r}}\right]=S_{o}(\Psi),
$$

where $S_{o}$ remains constant along the streamlines $\Psi=$ const. Equation (1.4) can be recast in the form

$$
c_{p}\left(T-T_{r}\right)-\frac{p-p_{r}}{\rho}=c_{p} T_{r} S_{o}(\Psi),
$$

and taking into account Bernouilli's equation $\left(p-p_{r}\right) / \rho+\left(v^{2}+w^{2}\right) / 2=\Pi(\Psi)$ and (1.5), one arrives at

$$
c_{p}\left(T-T_{r}\right)+\frac{1}{2}\left(v^{2}+w^{2}\right)=c_{p} T_{r} S_{o}(\Psi)+\Pi(\Psi)=H(\Psi),
$$

where $\Pi$ is constant along the streamlines. Finally from the definition of total temperature $T_{t}$,

$$
c_{p} T_{t}=c_{p} T+\frac{1}{2}\left(w^{2}+v^{2}\right)=c_{p} T_{r}+H(\Psi) ;
$$


note that the total temperature $T_{t}$ and total enthalpy $h_{t}=c_{p} T_{t}$ remain constant along the streamlines.

For the self-similar flows considered by Herrada et al. (1999), $H(\Psi)$ behaves near the axis as

$$
H(\Psi)=H_{o} r^{2(m-2)},
$$

so that (1.7) together with (1.1) and (1.9) lead to the following expression for the near-axis behaviour of the static temperature:

$$
T=T_{r}+\alpha \frac{(L W)^{2}}{2(m-2) c_{p}} r^{2(m-2)},
$$

where

$$
\alpha=\frac{(m-2)}{L^{2}}\left[\frac{2 H_{o}}{c_{p} W^{2}}-\left(1+L^{2}\right)\right]
$$

is a dimensionless constant which contains the arbitrary dimensional constant $H_{o}$ characterizing the total temperature field along the streamlines. Note that, in addition to exponent $m$, the inviscid and non-conducting self-similar motions considered by Herrada et al. are characterized by three integrals of the motion, $W, L$ and $H_{o}$ ( or $\alpha$ ), which correspond to Bernouilli, Kelvin, and stagnation enthalpy conservation theorems. Note also that for $0<m<2$, the stagnation temperature in the inviscid motion increases towards the axis if

$$
\alpha \leqslant(2-m)\left(1+1 / L^{2}\right), \quad \text { or equivalently } H_{o} \geqslant 0 .
$$

It is found that under condition (1.9) self-similar solutions to the thermal problem exist and, for relevant values of the parameters of the problem, they exhibit a substantial reduction of the total temperature near the vortex axis. The same effect of thermal separation has been observed in real vortex-jet cores to which the quasiincompressibility assumption applies to a first approximation, for instance the flow inside Ranque-Hilsch devices. In effect, typical values in Ranque-Hilsch tubes are: $\Delta T / T$ ranging from 0.1 to 0.2 , with $\Delta T \sim 30$ to $40 \mathrm{~K}$ and $T \sim 300 \mathrm{~K}$ and the characteristic Mach number of the flow in the interval $M^{2} \sim 0.3$ to 0.15 with velocities ranging from $100 \mathrm{~m} \mathrm{~s}^{-1}$ to $50 \mathrm{~m} \mathrm{~s}^{-1}$. Finally, buoyancy effects are neglected as a norm in the analysis of flows inside Ranque-Hilsch devices since the buoyancy to inertial force ratio is very small. For tube lengths ranging from 0.2 to $0.5 \mathrm{~m}$, the maximum order of the ratio between the characteristic buoyancy velocity and the velocity of the forced flow $[g H(\Delta T / T)]^{1 / 2} / v$ is typically $10^{-2}$.

Self-similar solutions at high Reynolds numbers have provided valuable information about both the mechanical (Fernández-Feria et al. 1995) and thermal (Herrada et al. 1999) aspects of the structure of vortex cores. However, the self-similar analysis is unable to provide any information about the evolution of the vortex since it is just concerned with the far downstream structure of the vortex after it has lost its memory of upstream conditions. Thus, questions such as whether a given vortex defined at a certain upstream station will actually evolve downstream towards a self-similar structure or, on the contrary, will break down, are beyond the scope of the self-similar analysis. By the same token, the physical mechanism leading to thermal separation cannot be elucidated by this kind of analysis. Therefore, in an attempt to answer these questions, we have computed numerically the mechanical and thermal downstream evolution of a vortex-jet core using the QC approximation to the Navier-Stokes equations. To investigate if the evolution leads to the self-similar state predicted by Fernández-Feria et al. (1995), and also Herrada et al. (1999) for the thermal case, we 


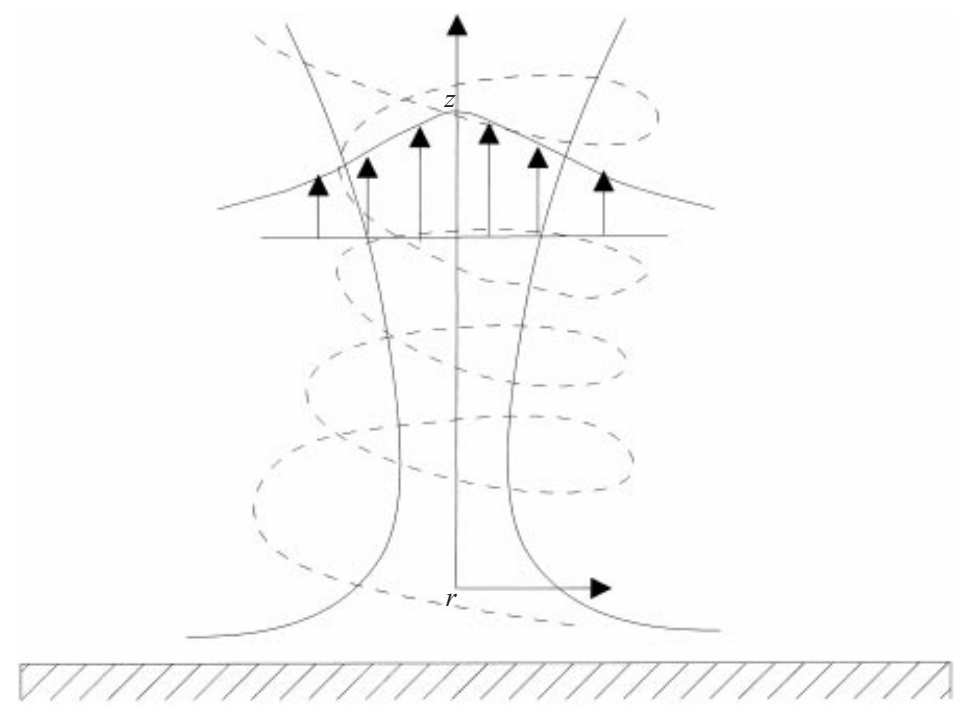

FIGURE 1. Sketch of the flow considered.

have imposed that the velocity, pressure and temperature fields very far from the axis match the external flow considered by those authors, namely the conical self-similar inviscid and non-conducting solutions to Euler equations. Therefore, the behaviour of the numerical solutions far from the axis must coincide with that of the self-similar Euler solutions near the axis ((1.1), (1.2), and (1.9)).

In addition to the outer flow conditions, characterized by parameters $m, L, W$ and $\alpha$, the evolution of a slender core also depends on the conditions at the upstream station $(z=0)$ at which the numerical integration starts. These initial conditions should be determined by solving the very complicated full (non-slender) set of Navier-Stokes equations in the region where the flow turns and gives rise eventually to the vortex jet, see figure 1. Nonetheless, since our interest is in the description of the downstream evolution of the resulting vortex jet, we have not considered the details of the flow in the turning region, but instead we have imposed at $z=0$ a velocity field which is a generalization of the Burger vortex. This type of initial condition generalizes to the case $m \neq 1$ the one used by Burggraf \& Foster (1977) in their numerical study of the downstream evolution of a vortex with $m=1$.

In our analysis, the upstream velocity profile is characterized by a dimensionless parameter $a$ which accounts for the axial momentum of the vortex jet at the upstream station. As a result of the study of the vortex evolution we will obtain the critical value of the swirl strength $L=L_{b}(a)$, as a function of $a$, beyond which any vortex with $L>L_{b}(a)$ must breaks down at a certain downstream station. Numerical results also show that the limit of the breakdown criterion $L_{b}(a)$ for large values of parameter a coincides with the breakdown criterion $L=L^{*}$ given by Fernández-Feria et al. (1995), beyond which self-similar vortex cores do not exist. Finally, our numerical study permits us to compute the total temperature profile $T_{t}(r)$ at several downstream stations as well as the different terms entering in the total temperature equation. In this way, we obtain a picture of the physical mechanism of thermal separation resulting from a self-consistent plausible model which, to the best of our knowledge, is lacking in the existing literature.

The paper is organized as follows. The equations and boundary conditions of the 
problem are given in $\S 2$. Section 3 contains a detailed description of the numerical procedure. The results obtained are summarized and discussed in $\S 4$. Section 5 contains conclusions. Finally, an analysis of the role of viscosity in the vortex breakdown in the flows considered in this paper is carried out in the Appendix.

\section{Equations and boundary conditions}

If the characteristic Reynolds number of the flow sketched in figure 1 is large enough, $R e=W \delta^{m-1} / v \gg 1$, where $W \delta^{m-2}$ is a characteristic velocity of the flow, $\delta$ is a characteristic vortex core length, and $v$ is the kinematic viscosity, which is assumed to be independent of the temperature, the effect of viscosity is only significant in two thin regions: the turning region near the wall plane and the vortex core. Outside these two regions we will assume that the axial and azimuthal velocity components of the inviscid flow decay far from the axis as $r^{m-2}$. In dimensionless cylindrical coordinates $(r, \phi, z)$, the QC equations governing the slender, viscous, quasi-incompressible vortexjet core are

$$
\begin{gathered}
\frac{1}{r} \frac{\partial}{\partial r}(r u)+\frac{\partial w}{\partial z}=0 \\
\frac{v^{2}}{r}=\frac{\partial p}{\partial r} \\
u \frac{\partial v}{\partial r}+w \frac{\partial v}{\partial z}+\frac{v u}{r}=\left[\frac{1}{r} \frac{\partial}{\partial r}\left(r \frac{\partial v}{\partial r}\right)-\frac{v}{r^{2}}\right], \\
u \frac{\partial w}{\partial r}+w \frac{\partial w}{\partial z}=-\frac{\partial p}{\partial z}+\left[\frac{1}{r} \frac{\partial}{\partial r}\left(r \frac{\partial w}{\partial r}\right)\right], \\
u \frac{\partial T}{\partial r}+w \frac{\partial T}{\partial z}=u \frac{\partial p}{\partial r}+w \frac{\partial p}{\partial z}+\frac{1}{P r}\left[\frac{1}{r} \frac{\partial}{\partial r}\left(r \frac{\partial T}{\partial r}\right)\right]+\left[\left(\frac{\partial w}{\partial r}\right)^{2}+\left(\frac{\partial v}{\partial r}-\frac{v}{r}\right)^{2}\right],
\end{gathered}
$$

where $p, T, u, v$ and $w$ are dimensionless and denote pressure, temperature (measured from the reference temperature $T_{r}$ ), and radial, azimuthal and axial components of the velocity respectively. To write equations (2.1)-(2.5) in dimensionless form we have used $\delta$ and $\operatorname{Re} \delta$ as radial and axial characteristic length scales, $W \delta^{m-2}$ as the characteristic velocity of the axial and azimuthal motions, $v / \delta$ as the radial characteristic velocity, $\rho W^{2} \delta^{2(m-2)}$ as the characteristic pressure, and $W^{2} \delta^{2(m-2)} / c_{p}$ as the characteristic temperature; $\rho$ is the fluid density, $P r=\rho v c_{p} / \lambda$ is the Prandtl number, and $\lambda$ is the thermal conductivity which will be taken as constant throughout the analysis.

Equations (2.1)-(2.5) must be solved subject to the boundary conditions

$$
\begin{gathered}
u=v=\partial w / \partial r=\partial T / \partial r=0 \quad \text { at } \quad r=0, \\
v \rightarrow L r^{m-2}, \quad w \rightarrow r^{m-2}, \quad p \rightarrow L^{2} r^{2 m-4} /(2 m-4) \quad \text { as } \quad r \rightarrow \infty
\end{gathered}
$$

and

$$
T \rightarrow \alpha \frac{L^{2}}{2(m-2)} r^{2 m-4} \quad \text { as } \quad r \rightarrow \infty .
$$

Note that it is only far from the axis $(r \rightarrow \infty)$ that the vortex flow is required to match the self-similar conical Euler solutions found by Fernández-Feria et al. (1995); of course, for finite values of $r$ the vortex structure will not, in general, be self-similar. 
In order to start the numerical integration of equations (2.1)-(2.5), the velocity and temperature profiles must be specified at some upstream station. Without entering into details of the flow in the turning region, see for example the sketch in figure 1, we have chosen as initial conditions at $z=0$ a generalized Burger's vortex-jet-like profile of the form

$$
\begin{gathered}
v_{i}=L r^{m-2}\left[1-\exp \left(-r^{3-m}\right)\right], \\
w_{i}=r^{m-2}\left[1-\exp \left(-r^{3-m}\right)\right]^{\frac{2-m}{3-m}}+(a-1) \exp \left(-r^{3-m}\right),
\end{gathered}
$$

where $a$ is the dimensionless axial velocity at the axis. Since we are primarily interested in explaining the mechanisms of the thermal separation which takes place in the downstream evolution of vortex cores, we will assume, for simplicity, that at the upstream station the stagnation temperatures of both the outer boundary and the core are uniform; this requires that in equations (1.7), (1.8) and (1.10)

$$
H_{o}=0 \quad \text { or equivalently } \alpha=(2-m)\left(1+1 / L^{2}\right) .
$$

In this case, the condition in $(2.8)$ is

$$
T \rightarrow-\frac{1}{2}\left(1+L^{2}\right) r^{2(m-2)} \quad \text { as } \quad r \rightarrow \infty .
$$

It is clear that this behaviour is met at the upstream station $z=0$, if one takes the temperature distribution in the core there to be

$$
T_{i}=-\frac{1}{2}\left(w_{i}^{2}+v_{i}^{2}\right) .
$$

Equations (2.1)-(2.5) and boundary conditions (2.6), (2.7) and (2.12), and (2.9), (2.10) and (2.13) show that the parameters governing the flow are the Prandtl number $P r$, Squire number $L$, exponent $m$, and parameter $a$; the latter characterizes the amount of axial momentum in the vortex core at the upstream station. In this work, we will consider only downstream evolution of vortex cores with Prandtl number $P r=0.72$. The influence of varying Prandtl number, including asymptotic, for both large and small values of $P r$, has been analysed for self-similar vortices by Herrada et al. (1999). Note that profiles (2.9), (2.10) and (2.13) satisfy conditions (2.6), (2.8) and (2.12) and include the initial velocity profiles considered by Burggraf \& Foster for $m=1$ as a particular case; in this case $(m=1) a$ plays the role of the flow force $M$.

The numerical integration of (2.1)-(2.6) and (2.7) and (2.12) determines the mechanical and thermal downstream evolution of the initial vortex (2.9), (2.10) and (2.13) once the values of $L, a$ and $m$ are specified. As we shall see later, the numerical results show that for a given $m$, the QC solutions break down at a finite distance from the upstream station for values of the parameters $L$ and $a$ lying on a certain region of the $(L, a)$-plane, while the integration of (2.1)-(2.8) and (2.12)-(2.13) can proceed smoothly and indefinitely for values of parameters $L$ and $a$ outside the breakdown region. In this last case, we found that the numerical solution of (2.1)-(2.8) and (2.12)-(2.13) tends for large $z$ to one of the self-similar solutions reported by Fernández-Feria et al. (1995) and Herrada et al. (1999). Unlike the work by Burggraf $\&$ Foster (1977) who studied the occurrence of breakdown in vortex jets characterized only by the flow force $a$, we have considered here a family of vortex jets defined not only by parameter $a$ but also by two additional parameters $L$ and $m$ which account for the swirl strength and for the rate of decay of the velocity field for large distance from the axis. 


\section{Numerical integration}

For the numerical integration of (2.1)-(2.6) and (2.12)-(2.13) we have employed an explicit line method. From the numerical point of view it is convenient to use the meridian stream function $\Psi$ and the circulation $\Gamma=r v$ as new dependent variables. In terms of the new variables, system (2.1)-(2.5) becomes

$$
\begin{gathered}
\frac{\Gamma^{2}}{r^{3}}=p_{r}, \\
-\frac{\Psi_{z} \Gamma_{r}}{r}+\frac{\Psi_{r} \Gamma_{z}}{r}=\Gamma_{r r}-\frac{\Gamma_{r}}{r}, \\
-\frac{\Psi_{z}}{r}\left(\frac{\Psi_{r}}{r}\right)_{r}+\frac{\Psi_{r}}{r}\left(\frac{\Psi_{r}}{r}\right)_{z}=-p_{z}+\left(\frac{\Psi_{r}}{r}\right)_{r r}+\frac{1}{r}\left(\frac{\Psi_{r}}{r}\right)_{r}, \\
-\frac{\Psi_{z}}{r} T_{r}+\frac{\Psi_{r}}{r} T_{z}=-\frac{\Psi_{z}}{r} \frac{\Gamma^{2}}{r^{3}}+\frac{\Psi_{r}}{r} p_{z}+\frac{1}{P r}\left(T_{r r}+\frac{1}{r} T_{r}\right) \\
+\left(\frac{\Psi_{r r}}{r}-\frac{\Psi_{r}}{r^{2}}\right)^{2}+\left(\frac{\Gamma_{r}}{r}-2 \frac{\Gamma}{r^{2}}\right)^{2},
\end{gathered}
$$

where subscripts $r$ and $z$ denote derivatives with respect to the radial and axial coordinates.

Eliminating the derivatives of pressure $p$ with respect to $z$ and $r$ from system (3.1)-(3.4), one arrives at

$$
\begin{gathered}
B_{1} \Psi_{z}+B_{2} \Psi_{z r}+B_{3} \Psi_{z r r}=B_{4}, \\
\Gamma_{z}=\frac{\Gamma_{r}}{\Psi_{r}} \Psi_{z}+\frac{r \Gamma_{r r}-\Gamma_{r}}{\Psi_{r}}, \\
T_{z}=\frac{\Psi_{z}}{\Psi_{r}} T_{r}-\frac{\Psi_{z}}{\Psi_{r}} \frac{\Gamma^{2}}{r^{3}}+\frac{\Psi_{z}}{r}\left(\frac{\Psi_{r}}{r}\right)_{r}-\frac{\Psi_{r}}{r}\left(\frac{\Psi_{r}}{r}\right)_{z}+\left(\frac{\Psi_{r}}{r}\right)_{r} r+\frac{1}{r}\left(\frac{\Psi_{r}}{r}\right)_{r} \\
+\frac{1}{P r}\left(\frac{r}{\Psi_{r}} T_{r r}+\frac{1}{\Psi_{r}} T_{r}\right)+\frac{r}{\Psi_{r}}\left[\left(\frac{\Psi_{r r}}{r}-\frac{\Psi_{r}}{r^{2}}\right)^{2}+\left(\frac{\Gamma_{r}}{r}-2 \frac{\Gamma}{r^{2}}\right)^{2}\right],
\end{gathered}
$$

where functions $B_{1}, B_{2}, B_{3}$ and $B_{4}$ are

$$
\begin{gathered}
B_{1}=-r^{2} \Psi_{r r r}+3 r \Psi_{r r}-3 \Psi_{r}+2 r \Gamma \Gamma_{r} / \Psi_{r}, \\
B_{2}=-r \Psi_{r}, \quad B_{3}=r^{2} \Psi_{r}, \\
B_{4}=r^{3} \Psi_{r r r r}-2 r^{2} \Psi_{r r r}+3 r \Psi_{r r}-3 \Psi_{r}-2 \Gamma\left(r \Gamma_{r r}-\Gamma_{r}\right) / \Psi_{r} .
\end{gathered}
$$

The discretization of (3.5)-(3.7) into $N+1$ points along the radial direction $\left[r_{i}=(i-1) \mathrm{d} r, i=1 \ldots N+1, \mathrm{~d} r=r_{\infty} /(N-1)\right]$ allows efficient integration of them. Using second-order central differences, one then obtains a system of $3 N-3$ ordinary differential equations of the form

$$
\boldsymbol{A}\left(\begin{array}{c}
\Psi_{z}(1) \\
\vdots \\
\Psi_{z}(N-1)
\end{array}\right)=\boldsymbol{R}_{1}, \quad\left(\begin{array}{c}
\Gamma_{z}(1) \\
\vdots \\
\Gamma_{z}(N-1)
\end{array}\right)=\boldsymbol{R}_{2}, \quad\left(\begin{array}{c}
T_{z}(1) \\
\vdots \\
T_{z}(N-1)
\end{array}\right)=\boldsymbol{R}_{3},
$$


where $\boldsymbol{A}$ is the tridiagonal matrix:

$$
\boldsymbol{A}=\left[\begin{array}{ccccccc}
b_{1} & c_{1} & 0 & \cdots & & & \\
a_{2} & b_{2} & c_{2} & \cdots & & & \\
& & & \ddots & & & \\
& & & \cdots & a_{N-2} & b_{N-2} & c_{N-2} \\
& & & \cdots & 0 & a_{N-1} & b_{N-1}
\end{array}\right],
$$

with coefficients $a_{i}, b_{i}$ y $c_{i}(i=1, \ldots, N-1)$, given by

$$
\begin{aligned}
a_{i} & =-B_{2}\left(r_{i}, z\right) /(2 \mathrm{~d} r)+B_{3}\left(r_{i}, z\right) / \mathrm{d} r^{2}, \\
b_{i} & =B_{1}\left(r_{i}, z\right)-2 B_{3}\left(r_{i}, z\right) / \mathrm{d} r^{2}, \\
c_{i} & =B_{2}\left(r_{i}, z\right) /(2 \mathrm{~d} r)+B_{3}\left(r_{i}, z\right) / \mathrm{d} r^{2},
\end{aligned}
$$

and vectors $\boldsymbol{R}_{1}, \boldsymbol{R}_{2}, \boldsymbol{R}_{3}$ are given by

$$
\begin{gathered}
R_{1}(i)=B_{4}\left(r_{i}, z\right), \\
R_{2}(i)=\left(\frac{\Gamma_{r}}{\Psi_{r}} \Psi_{z}+\frac{r \Gamma_{r r}-\Gamma_{r}}{\Psi_{r}}\right)_{\left(r_{i}, z\right),} \\
R_{3}(i)=\left\{\frac{\Psi_{z}}{\Psi_{r}} T_{r}-\frac{\Psi_{z}}{\Psi_{r}} \frac{\Gamma^{2}}{r^{3}}+\frac{\Psi_{z}}{r}\left(\frac{\Psi_{r}}{r}\right)_{r}-\frac{\Psi_{r}}{r}\left(\frac{\Psi_{r}}{r}\right)_{z}+\left(\frac{\Psi_{r}}{r}\right)_{r r}+\frac{1}{r}\left(\frac{\Psi_{r}}{r}\right)_{r}\right. \\
\left.+\frac{1}{P r}\left(\frac{r}{\Psi_{r}} T_{r r}+\frac{1}{\Psi_{r}} T_{r}\right)+\frac{r}{\Psi_{r}}\left[\left(\frac{\Psi_{r r}}{r}-\frac{\Psi_{r}}{r^{2}}\right)^{2}+\left(\frac{\Gamma_{r}}{r}-2 \frac{\Gamma}{r^{2}}\right)^{2}\right]\right\}\left(r_{i}, z\right) .
\end{gathered}
$$

In terms of $\Psi, \Gamma$ and $T$ the initial conditions (2.9)-(2.13) and the boundary conditions (2.6)-(2.8) and (2.12) are

$$
\begin{aligned}
\Psi_{r}(r, 0) & =r^{m-1}\left[1-\exp \left(-r^{3-m}\right)\right]^{\frac{2-m}{3-m}}+(a-1) r \exp \left(-r^{3-m}\right), \\
\Gamma(r, 0) & =L / r^{1-m}\left[1-\exp \left(-r^{3-m}\right)\right], \\
T(r, 0) & =-\frac{1}{2}\left(\Gamma^{2}(r, 0) / r^{2}+\Psi_{r}^{2}(r, 0) / r^{2}\right),
\end{aligned}
$$

and

$$
\begin{gathered}
\Psi=\Gamma=\Psi_{r}=T_{r}=0 \quad \text { on } \quad r=0, \\
\Gamma \rightarrow L r^{m-1}, \quad \Psi_{r} \rightarrow r^{m-1}, \quad T \rightarrow-\frac{1}{2}\left(L^{2}+1\right) r^{2 m-4} \quad \text { as } \quad r \rightarrow r_{\infty} .
\end{gathered}
$$

Since tridiagonal systems can be very easily solved numerically (Press et al. 1989), equations (3.5)-(3.7) with conditions (3.18)-(3.22) can be efficiently integrated in the $z$-direction using a variable-step-size fourth-order Runge-Kutta method once $m, a$ and $L$ are specified. In our calculations the number of lines $N=2600$ have been used to discretize the radial variable. Moreover, the boundary of the numerical domain has been taken sufficiently far from the axis, $r_{\infty}=300$, to ensure that the vortex core, which grows downstream, always remains inside the considered domain. The numerical accuracy of the code has been tested repeatedly for several values of $N$. Results are insensitive up to the fifth digit for $N>2600$. Note that our procedure permits automatic control of the error for the downstream integration along the lines ( $z$-direction) by the Runge-Kutta adaptive step size; the relative error tolerance given to the 


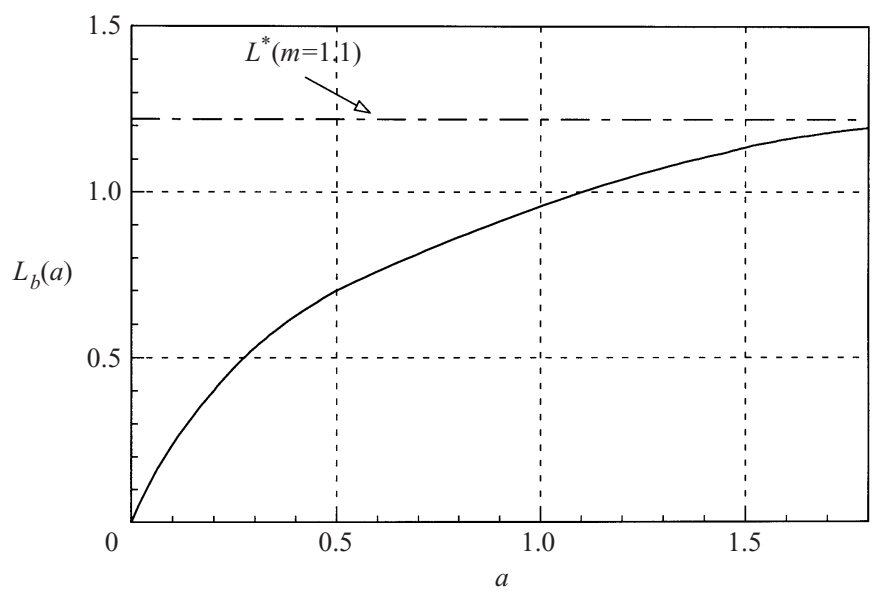

Figure 2. Critical values $L_{b}$ as a function of $a$. The critical self-similar value $L^{*}(m=1.1)$ is also given.

Runge-Kutta solver was $10^{-5}$. Note also that the use of the method of lines avoids the complicated and expensive nonlinear iteration required by finite difference methods.

\section{Numerical results}

\subsection{Mechanical problem}

Ignoring perturbations of finite amplitude, the question of whether a given vortex breaks down or remains slender indefinitely downstream can be answered by solving the problem formulated in $\S \S 2$ and 3. Numerical results show that for given values of parameters $a$ and $m$, there exists a critical value $L_{b}(a, m)$ such that if $L<L_{b}$ the numerical integration may proceed downstream arbitrarily far, while if $L>L_{b}$ the slender structure of the vortex breaks up at a certain value of $z$ giving rise to a non-slender structure. In the former case, we find that the vortex jet evolves towards the self-similar solution corresponding to the given values of $L$ and $m$ as described by Fernández-Feria et al. (1995). Figure 2 shows values of $L_{b}$ as a function of parameter $a$ for $m=1.1$. It is found that $L_{b}$ grows monotonically with $a$, and has an asymptote at $L_{b}=L^{*}(m)$ which is precisely the breakdown threshold given by Fernández-Feria et al. (1995). This threshold value $L^{*}$, which was obtained by these authors from the condition of non-existence of similarity solutions, provides a conservative criterion for breakdown for the evolution of non-self-similar vortices like those considered here, since, independently of the value of $a$, a vortex jet with a value of $L$ larger than $L^{*}(m)$ must break up at a certain distance downstream. Figure 3 shows the velocity at the axis as a function of the downstream distance $z$ for three vortices characterized by different values of parameters $L$ an $a$ but the same value of $m,(m=1.1)$. It can be seen that, in the case $L=1.5$ and $a=2.5$, the velocity at the axis decreases abruptly and the QC approximation fails at the station $z=z^{*} \simeq 1.9$, where vortex breakdown takes place. Note that independently of the value of parameter $a$, a vortex with $L=1.5$ and $m=1.1$ will unavoidably break since the vortex breakdown threshold $L^{*}(m=1.1) \simeq 1.22$ (see figure 2) is smaller than $L=1.5$; of course, the distance $z^{*}$ at which the vortex breaks down increases with $a$. The other two vortices considered in figure 3 have the same value of $L, L=1.2$, but different values of $a$. The vortex with less velocity at the upstream station, $a=1.6$, breaks at $z^{*} \simeq 0.79$ since, in this case, 


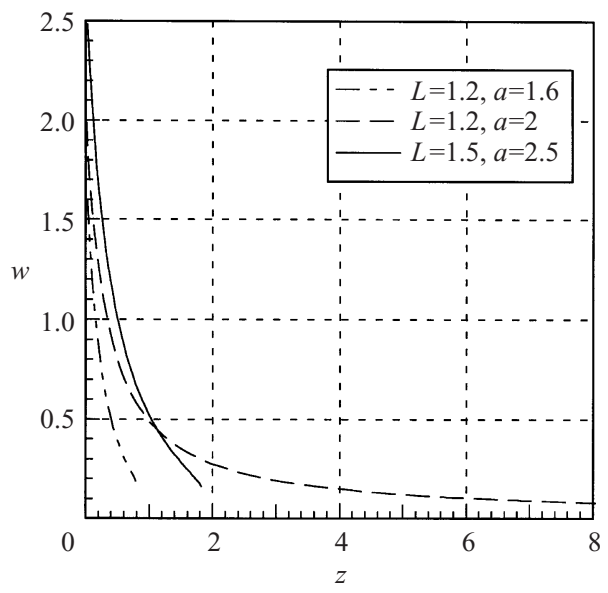

FIGURE 3. Axial velocity at the axis as a function of $z$ for $m=1.1$ and different values of parameters $a$ and $L$.

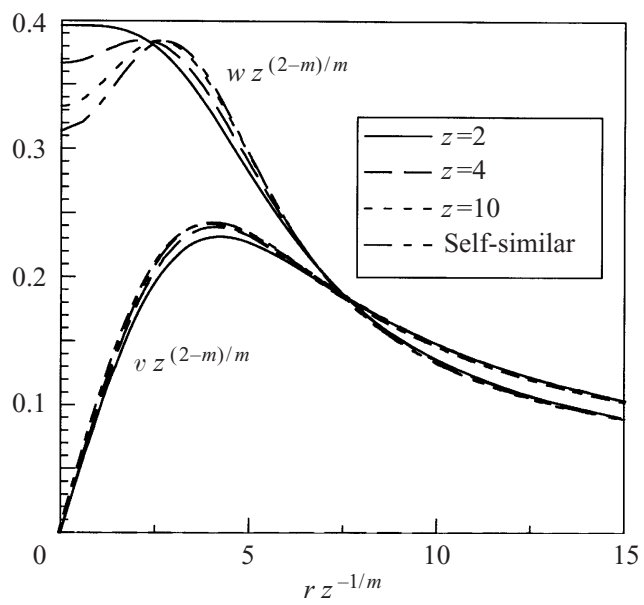

FIGURE 4. Normalized axial $w z^{(2-m) / m}$ and azimuthal $v z^{(2-m) / m}$ velocities for a vortex with $m=1.1$, $a=1.9, L=1.2$ as a function of the normalized radial distance $r / z^{1 / m}$ at different $z$-stations. Self-similar velocity profiles are also given for comparison.

the breakdown value $L_{b}(a=1.6) \simeq 1.15$ (see figure 2 ) is smaller than $L=1.2$. On the contrary, the vortex with $a=2$ evolves towards a self-similar solution since $L=1.2$ is smaller than the breakdown value $L_{b}(a=2) \simeq 1.21$.

Figure 4 shows the computed axial and azimuthal velocity profiles of a vortex with $L=1.2$ and $a=1.9$ at three different downstream stations $(z=2, z=4$, and $z=10$ ). The velocity and the radial distance have been appropriately re-scaled for comparison to the self-similar solution, which is also plotted in the figure. Note that the vortex considered evolves towards a self-similar solution since in this case $L=1.2<L_{b}(a=1.9)$. Note also that due to the action of centrifugal forces the axial velocity profile changes from jet-like to wake-like.

Before closing this section, let us point out that in associating vortex breakdown with the failure of the QC approximation at some downstream station, we are following Hall's approach to the breakdown phenomenon. Of course, we do not 
mean that the failure of the $\mathrm{QC}$ equations provides a physical explanation of the phenomenon: that, on the contrary, must be sought in the full (non-slender) NavierStokes equations. In fact, what appears as a catastrophic event from the point of view of the QC equations must be a sharp, but continuous, transition from a slender to a non-slender flow when described with the full Navier-Stokes equations. Clearly, it is in this transition region where the physical explanation to vortex breakdown must be sought. In any case, there is little question that the QC equations remain a valuable tool in the analysis of many real vortex flows, since the QC results concerning the downstream evolution of the vortex up to very near the breakdown station are rather close to the ones found by sophisticated numerical models and experiments. Therefore, although the QC failure does not provide a complete physical explanation to vortex breakdown, it can be seen as a symptom which accompanies breakdown and gives important clues about the role that viscosity plays in that phenomenon. In effect, our results, and those from self-similar analysis, suggest that viscosity plays a very important role in the breakdown of the unconfined vortices considered here. In fact, it seems that if the swirl strength exceeds the critical value $L_{b}(a, m)$, the viscous axial momentum transfer from the region of maximum axial velocity is not efficient enough to keep the vortex confined in a slender configuration against the effect of the centrifugal forces. Then the radial velocity suddenly increases and, as required by continuity, the axial velocity decreases and the vortex breaks down. This scenario suggests that in vortex flows where viscosity is the mechanism which regularizes the behaviour of the velocity field near the axis, viscous effects must play an essential role in the breakdown phenomenon. To illustrate this, we have considered a family of inviscid self-similar vortex-jet flows with a singular behaviour at the axis. As shown in the Appendix, when Benjamin criterion is applied to this family, it is found that it does not exhibit breakdown for any value of $L$. However if, to simulate a real jet vortex, one tries to regularize the flow by a slender viscous vortex core which far from the axis matches the given inviscid family, one finds that such a slender viscous core exists only if $L$ is less than a critical value.

\subsection{Thermal problem}

Numerical results from the energy equation yield both the total and static temperature fields. Radial profiles of the total temperature $T_{t}$ in the vortex core for several values of the downstream distance $z$ are plotted in figure 5. The total temperature, which at the upstream station $(z=0)$ is uniform along the radial direction, evolves towards a non-uniform profile with lower values of the total temperature in the near-axis zone and higher ones far from the axis.

To elucidate the physical mechanisms leading to the decrease of the total temperature in swirling flows, it is convenient to write the equation for the dimensionless total temperature $T_{t}$ in the form

$$
w \frac{\partial T_{t}}{\partial z}=-u \frac{\partial T_{t}}{\partial r}+P r^{-1} \nabla^{2} T+\tau: \nabla v+v \cdot \nabla \cdot \tau,
$$

where $\tau$ and $\boldsymbol{v}$ represent the viscous stress tensor and the velocity field respectively. Equation (4.1) expresses the convection of total temperature in the axial direction as a result of convection of total temperature in the radial direction, heat conduction, viscous dissipation and the mechanical work of viscous forces respectively. For the vortex jet considered in figure 5 , we have computed, at a given station $(z=0.1)$, the QC approximation of each of the terms in (4.1) and the results are plotted in figure 6. As shown in the figure, in the near-axis region the total temperature decreases in 


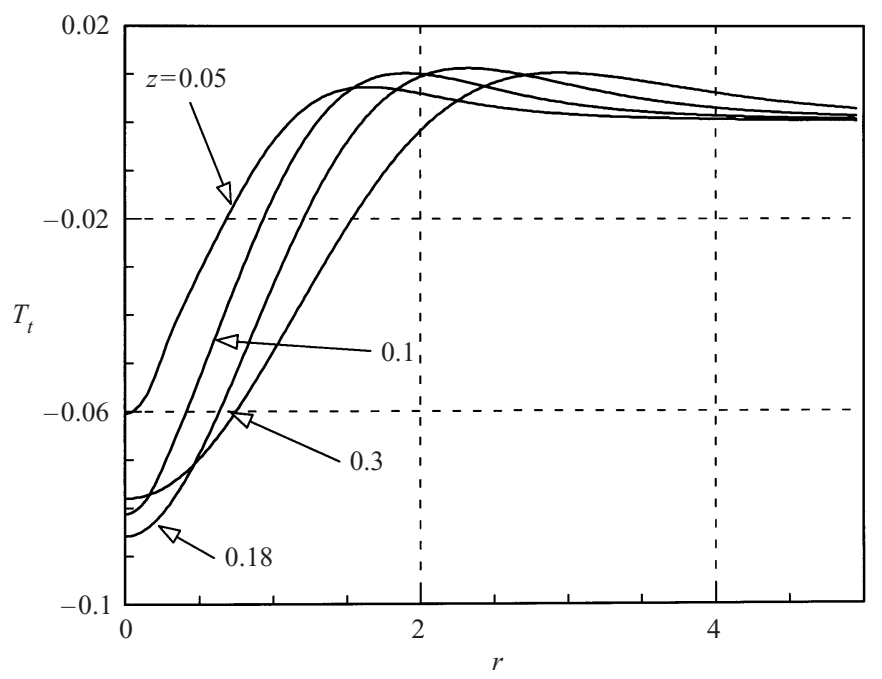

FiguRE 5. Total temperature profiles at different $z$-stations for $m=1.1, a=1.35, L=1.079$, $\operatorname{Pr}=0.72$

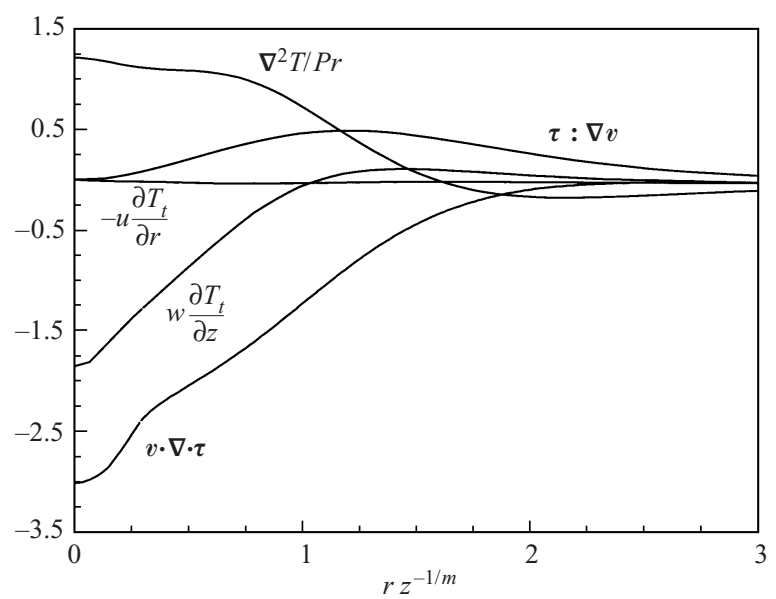

FIGURE 6. Illustration of the physical mechanisms involved in thermal separation. Values have been computed at the station $z=0.1$ for the vortex jet considered in figure 5 .

the axial direction since $w \partial T_{t} / \partial z<0$ and $w>0$. This decrease in total temperature (separation effect) is due to the intense loss of kinetic energy near the axis as a result of the mechanical work of viscous forces (the only term which gives a negative contribution to the right-hand side of (4.1)) since both heat conduction and viscous dissipation tend to increase the total temperature in the near-axis zone, and the effect of radial convection is almost negligible. It should be noticed that diffusion effects, both viscous and heat conduction, are essential to the thermal separation phenomenon since, otherwise, the total temperature of the fluid particles remains constant along the streamlines.

It can be observed in figure 5 that the minimum value of the total temperature, which is located at the axis, first decreases downstream, reaches an absolute minimum at a certain station $(z \simeq 0.18$ for the case considered in figure 5) and then increases 


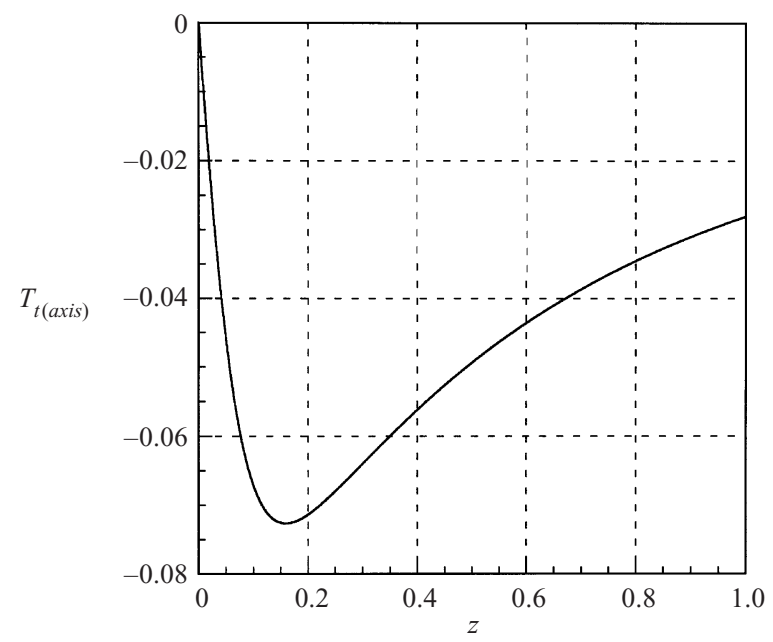

FIGURE 7. Total temperature at the axis as a function of $z$ for the vortex jet considered in figure 5 .

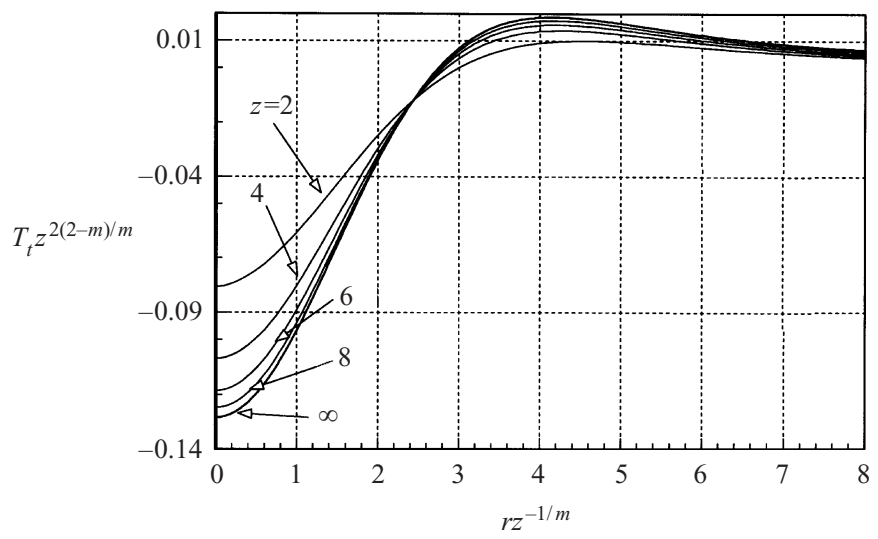

FIGURE 8. Normalized total temperature $T_{t} z^{(4-2 m) / m}$ as a function of the normalized radial distance $r / z^{1 / m}$ at different $z$-stations for $m=1.2, a=2, L=1.079, \mathrm{Pr}=0.72$. Self-similar profiles of the total temperature are also given for comparison.

steadily. This behaviour is plotted in figure 7 where values of the minimum total temperature at the axis as a function of $z$ are plotted. The abrupt decrease of the total temperature at the beginning of the downstream evolution is related to the strong slowing down of by the vortex. Beyond the station at which the absolute minimum in total temperature is reached, the increase in the static temperature due to heat conduction and viscous dissipation is greater than the loss of kinetic energy due to the slowing down of the vortex and, consequently, the stagnation temperature starts to increase with the axial distance.

Total temperature profiles at different $z$-stations are plotted in figure 8 for a vortex characterized by the values $m=1.2, a=2, L=1.075$, and $P r=0.72$. In this case, the vortex core evolves towards a self-similar solution and the variables have been appropriately re-scaled for easier comparison. As shown by Herrada et al. (1999), the decrease in total temperature (thermal separation) predicted by self-similar solutions is in good agreement with the observed values in real swirling flows.

The amount of thermal separation depends strongly on the swirl strength $L$ as 


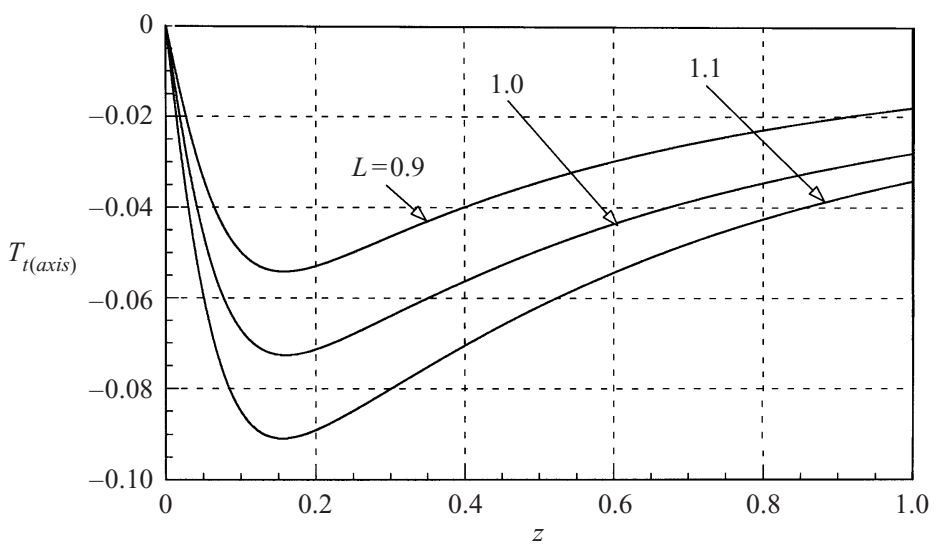

FIGURE 9. Total temperature at the axis as a function of $z$ for three different values of $L$ and $m=1.1, a=1.35, \operatorname{Pr}=0.72$.

shown in figure 9 where the downstream evolution of the total temperature at the axis is plotted for several values of $L$ and fixed values of the remaining parameters. Note that the temperature decrease for $L=1.1$ is almost twice that for $L=0.9$. This can be explained by noting that the larger the swirl strength the larger the nonfavourable pressure gradient along the $z$-direction $(\partial p / \partial z$ increases) and the larger the rate of loss of kinetic energy during the initial stages of the evolution. Observe also that after the station of minimum total temperature is reached, the rate of gain of static temperature by the action of heat conduction and viscous dissipation is greater than the rate of loss of kinetic energy by the slowing down effect of the viscous forces. This results in a net increase of total temperature as $z$ increases.

Finally, note that for the vortices considered in figure 9, the value $L=1.1$ is slightly less than the breakdown value $L_{b}(a=1.35)$ (figure 2) and, therefore, the amount of thermal separation for the case $L=1.1$ is very close to the maximum one that can be achieved if we demand that the vortex jet remains slender up to $z \rightarrow \infty$. Nonetheless, the value of $L$ and consequently the amount of thermal separation can be increased if this last condition is relaxed by just requiring that the vortex remains slender up to a certain station beyond that where the minimum of total temperature is reached. This increase of thermal separation should be of value in the design of Ranque tubes.

\section{Conclusions}

In this paper, the QC approximation has been used to study numerically both the mechanical and thermal downstream evolution of a viscous vortex-jet core driven by an inviscid but vortical flow whose velocity, pressure and temperature fields behave near the axis as

$$
v \rightarrow L r^{m-2}, \quad w \rightarrow r^{m-2}, \quad p \rightarrow L^{2} r^{2 m-4} /(2 m-4), \quad T \rightarrow-\frac{1}{2}\left(1+L^{2}\right) r^{2(m-2)} .
$$

The reason for solving the problem using the QC approximation is the drastic simplification which it introduces compared to the numerical solution of the complete Navier-Stokes equations, which is particularly relevant for very complicated open flows as those considered here. In addition, as Beran \& Culick (1992) have shown for the much simpler case of swirling flows in pipes, the QC results are expected to be close to the real ones. 
The swirling parameter $L$, defined as the ratio between the azimuthal and axial components of the inviscid velocity near the axis, is a characteristic of the outer inviscid swirling flow. In addition to $L$, the evolution of the vortex jet core depends on the velocity profile at the station at which we start the numerical integration. At this station, a generalized Burger's vortex-jet-like profile characterized by the dimensionless velocity at the axis $a$ has been considered. For given values of the parameters of the problem $(L, a$ and $m$ ), the numerical integration proceeds starting with profiles (2.9), (2.10) and (2.13) to obtain the downstream evolution of the velocity and temperature fields.

Numerical results show that for a given value of $m$, there exists a critical value of the swirling parameter $L_{b}(a)$ which depends on $a$ such that if $L$ is smaller than $L_{b}$ the vortex core remains slender and its structure evolves asymptotically for large values of $z$ towards a self-similar one of the class described by Fernández-Feria et al. (1995). For $L$ larger than $L_{b}$ the vortex breaks up at a certain station $z^{*}$ and gives rise to a non-slender structure; the value of the breakdown distance $z^{*}$ increases for increasing values of $a$. We have also found that for a given $m$, the breakdown curve $L_{b}(a, m)$ has an asymptote $L^{*}(m)$ which coincides with the values found by Fernández-Feria et al. (1995) beyond which self-similar solutions fail to exist and which they identified with a criterion for vortex breakdown. In fact, for the kind of vortices considered here, $L^{*}$ can be taken as a conservative threshold for breakdown since a vortex jet with a value of $L$ larger than $L^{*}(m)$ breaks unavoidably at a certain distance downstream.

As previously suggested by the self-similar analysis, our numerical results confirm that the evolution of flows with a viscous vortex core cannot remain indefinitely slender for values of the swirl strength larger than a critical one. In these flows, viscous effects play a crucial role in vortex breakdown since they set limits to the values of $L$ which allow the existence of slender vortex-jet cores which are regular at the axis. In contrast, as shown in the Appendix, inviscid vortex-jet flows whose velocity field is singular at the axis do not present vortex breakdown for any value of $L$. This result confirms the important role that viscosity must play in the breakdown of real flows with a viscous vortex core. The quasi-incompressible QC equations provide a self-consistent model to compute the total temperature field as a function of the parameters of the problem and shed light on the physical mechanisms responsible for the phenomenon of thermal separation observed in real vortices (for example those in Ranque tubes).

Results of the thermal downstream evolution of the vortex core reveal a strong decay of the total temperature during the initial stages of the evolution; the thermal separation mechanism is mainly due to the loss of kinetic energy associated with the slowing down of the vortex core due to the mechanical work of the viscous forces. Once the viscous core has been substantially slowed down and the total temperature reaches a minimum, the effect of heat conduction and viscous dissipation dominates that of the mechanical work of viscous forces and the total temperature increases downstream due to the increase of the static temperature.

We have found a sharp dependence of the magnitude of the separation effect on parameter $L$ that can be attributed to the fact that the increase of centrifugal forces with $L$ gives rise to a stronger deceleration of the flow during the initial stages of the evolution.

This work has been partially supported by the Dirección General de Enseñanza Superior of Spain, PB96-0679-C02-02. 


\section{Appendix}

In this Appendix, we will apply Benjamin's theory to establish a criterion for breakdown of the inviscid family of conical vortices given by

$$
\Psi=s^{m} F(x, \xi), \quad C=K \Psi^{(m-1) / m}, \quad H=\frac{K_{1}}{2(2-m)} \Psi^{2(m-2) / m}, \quad \xi=\ln \left(s / s_{o}\right),
$$

where $(s, x=\cos \theta, \phi)$ are spherical coordinates, $s$ is the distance from the origin and $\theta$ and $\phi$ are the polar and azimuthal angles, $m$ is a real number in the range $1 \leqslant m<2$, and $K$ and $K_{1}$ are arbitrary positive dimensional constants related to the strength of the azimuthal and meridional motion respectively.

The stream function $\Psi$ satisfies the Bragg-Hawthorne equation (also known as the Long-Squire equation) which in spherical coordinates is

$$
\frac{\partial^{2} \Psi}{\partial s^{2}}+\frac{1-x^{2}}{s^{2}} \frac{\partial^{2} \Psi}{\partial x^{2}}=s^{2}\left(1-x^{2}\right) \frac{\mathrm{d} H}{\mathrm{~d} \Psi}-C \frac{\mathrm{d} C}{\mathrm{~d} \Psi},
$$

where the two Lagrangian constants of the motion, circulation $C$ and stagnation pressure $H$ defined as

$$
C=\oint \boldsymbol{v} \cdot \mathrm{d} \boldsymbol{l} \text { and } H=\frac{p}{\rho}+\frac{1}{2} \boldsymbol{v} \cdot \boldsymbol{v}
$$

depend only on $\Psi$. Expressions (A 1) represent mathematically the high Reynolds number flow inside the conical domain $\left(1 \geqslant x \geqslant x_{c}, s_{i} \leqslant s \leqslant s_{o}\right)$.

To analyse the spatial stability of these basic solutions we consider the following expansion:

$$
\Psi(s, x, \xi)=s^{m} F(x, \xi)=s^{m}\left[F_{b}(x)+F_{d}(x) \exp (\alpha \xi)+\cdots\right],
$$

where subscripts $b$ and $d$ denote basic and disturbance solutions and the eigenvalues $\alpha=\alpha_{r}+\mathrm{i} \alpha_{i}$ depend on the values $K$ and $K_{1}$ characterizing the basic solution. Following Benjamin (1962), basic flows with values of $K$ and $K_{1}$ which allow the existence of standing waves of zero frequency, $\alpha_{i} \neq 0$, are called subcritical while supercritical ones are those with values of $K$ and $K_{1}$ leading to real eigenvalues. Transition from supercritical to subcritical flows is interpreted as vortex breakdown.

On introducing (A 4) into (A 2) and (A 3) and defining

$$
Y=(m / K)^{2} F_{b}^{2 / m}, \quad D=2 m^{2} K_{1} / K^{4},
$$

we arrive, after neglecting terms of higher order, at the following two basic and disturbed problems:

$$
Y Y^{\prime \prime}+\frac{m-2}{2} Y^{\prime 2}+2 \frac{m-1}{1-x^{2}}\left(Y^{2}+Y\right)+D=0,
$$

with boundary conditions

$$
Y(1)=Y\left(x_{c}\right)=0,
$$

and

$$
\begin{gathered}
\left(1-x^{2}\right) F_{d}^{\prime \prime}+q(x, m, D, \alpha) F_{d}=0, \\
q=\left[(m-1+\alpha)(m+\alpha)+\frac{(m-4) D\left(1-x^{2}\right)}{2 Y^{2}}+\frac{(m-1)(m-2)}{Y}\right]
\end{gathered}
$$

with boundary conditions

$$
F_{d}(1)=F_{d}\left(x_{c}\right)=0
$$


Constant $D$ measures the strength of meridional motion $K_{1}$ relative to the strength of swirling motion $K$ and is related to the swirling parameter $L$ by

$$
D=2(2-m)\left(L^{2}+\frac{1-m}{4-2 m}\right)^{2}-\frac{(m-1)^{2}}{2(2-m)} .
$$

The basic problem (A 6)-(A 7) was considered in Fernández-Feria et al. (1999). It can be easily shown that solutions of equation (A 6) satisfying the boundary condition at the axis, $x=1$, behave as

$$
Y=2 a_{1}(1-x)\left(1+A(1-x)^{\lambda}\right)+O(1-x)^{2}, \quad x \rightarrow 1,
$$

where $a_{1}$ and $\lambda$ are the positive roots of

$$
(m-2) a_{1}^{2}+(m-1) a_{1}+D / 2=0 \quad \text { and } \quad \lambda^{2}+(m-1) \lambda+m-2+(m-1) /\left(2 a_{1}\right)=0 .
$$

Taking into account (A 5) and (A 12) it can be seen that (A 4) behaves near the axis as

$$
\Psi(s, 1) \simeq s^{m} \theta^{m} \simeq r^{m},
$$

which matches the behaviour given in (1.1) when $\theta \rightarrow 0$.

For given values of $m$ in the range $1 \leqslant m<2$, and of $x_{c}, D$ and $A$, one must start the numerical integration of (A 6) near the axis with behaviour (A 12) and proceed to the station $x_{c}$ where $Y\left(x_{c}\right)$ will not in general vanish. The numerical procedure has to be repeated with different values of $A$ until one is found for which the numerical solution of (A 6) satisfies the boundary condition $Y\left(x_{c}\right)=0$.

Problem (A 8)-(A 11) has two families of eigenvalues $\alpha^{k}$ and $\alpha_{k}(k=1,2, \ldots \infty)$ which are symmetric with respect to $\alpha=(1-2 m) / 2$ since they satisfy the condition $\alpha^{k}+\alpha_{k}=(1-2 m) / 2$. For given values of $m$ and $x_{c}$, and any arbitrary value of $D$ one finds numerically that both families are real and satisfy the condition $\alpha^{k} \alpha_{k}<0$ for any value of the index $k$. Moreover, the signs of $\alpha^{k}$ and $\alpha_{k}$ remain unchanged for $D$ ranging from zero to infinity. Accordingly, in the conical domain $s_{i} \leqslant s \leqslant s_{o}$, $x_{c} \leqslant x \leqslant 1$, a general steady perturbation induced by disturbances at the boundaries $\left(s=s_{i}, s=s_{o}\right.$ ) of a basic solution characterized by a value of $D$ outside the breakdown parametric region (supercritical basic flow) can be represented by superposition of modes with $\alpha_{k}<0$ (decaying for $\xi>0$ ) which provide the contribution of disturbances coming from the inner boundary $s=s_{i}$ and modes with $\alpha^{k}>0$ (decaying for $\xi<0$ ) which account for the contribution of disturbances from the outer boundary $s=s_{o}$. Therefore, perturbations can grow neither downstream nor upstream. Observe also that the supercritical character of the basic flow can never change as we vary $D$ (or $L$ ) since the sign of the eigenvalues are independent of $D$. Hence, according to the inviscid analysis, the family of vortex flows considered here with any arbitrary value of $L$ ranging from zero to infinity do not undergo vortex breakdown.

\section{REFERENCES}

Benjamin, T. B. 1962 Theory of vortex breakdown phenomenon. J. Fluid Mech. 14, 593-629.

Beran, P. S. \& Culick, F. E. C. 1992 The role of non-uniqueness in the development of vortex breakdown in tubes. J. Fluid Mech. 242, 491-527.

Burggraf, O. R. \& Foster, M. R. 1977 Continuation or breakdown in tornado-like vortices. J. Fluid Mech. 80, 685-703.

Deissler, R. G. \& Perlmutter, M. 1960 An analysis of flow and energy separation in a turbulent vortex. Intl J. Heat Mass Transfer 1, 173.

Emanuel, K. A. 1991 The theory of hurricanes. Annu. Rev. Fluid Mech. 23, 179-196. 
Escudier, M. P. 1988 Vortex breakdown: observations and explanations. Prog. Aero. Sci. 25, 189-229.

Fernández-Feria, R. 1999 Non-parallel stability analisis of Long's vortex. Phys. Fluids 11, 11141126.

Fernández-Feria, R., Fernández de la Mora, J. \& Barrero, A. 1995 Solution breakdown in a family of self-similar nearly inviscid axisymmetric vortices. J. Fluid Mech. 305, 77-91.

Fernández-Feria, R., Fernández de la Mora, J., Pérez-Saborid, M. \& Barrero, A. 1999 Conically similar swirling flows at high Reynolds numbers. Qt. J. Mech. Appl. Maths 52, 1-53.

Hall, M. G. 1972 Vortex breakdown. Annu. Rev. Fluid Mech. 4, 195-218.

Herrada, M. A., PÉrez-SAborid, M. \& Barrero, A. 1999 Thermal separation in near-axis boundary layers with intense swirl. Phys. Fluids 11, 3678-3687.

Keller, J. J., Egli, W. \& ExLey, J. 1985 Force and loss-free transition between flow states. Z. Angew. Math. Phys. 36, 854-889.

KurosaKa, M. 1982 Acoustic streaming in swirling flow and the Ranque-Hilsch effect. J. Fluid Mech. 124, 139-172.

Leibovich, S. 1984 Vortex stability and breakdown: survey and extension. AIAA J. 22, 1192-1206.

LoNG, M. G. 1961 A vortex in an infinite viscous fluid. J. Fluid Mech. 11, 611-623.

Ogawa, A. 1993 Vortex Flows. CRC Press.

Press, W. H., Flannery, B. P., Teukolski, S. A. \& Vetterling, W. T. 1989 Numerical Recipies. Cambridge University Press.

SARPKAYA, T. 1971 On stationary and travelling vortex breakdowns. J. Fluid Mech. 45, 545-559.

SARPKAYA, T. 1995 Turbulent vortex breakdown. Phys. Fluids 7, 2301-2303.

Shtern, V. \& Hussain, F. 1996 Hysteresis in swirling jets. J. Fluid Mech. 309, 1-44.

Vatistas, G. H., Lin, S. \& KwoK, C. K. 1986 Theorical and experimetal studies on vortex chambers flows. $A I A A$ J. 24, 635-842. 